This item was submitted to Loughborough's Research Repository by the author.

Items in Figshare are protected by copyright, with all rights reserved, unless otherwise indicated.

\title{
Null particle solutions in three-dimensional (anti-) de Sitter spaces
}

PLEASE CITE THE PUBLISHED VERSION

LICENCE

CC BY-NC-ND 4.0

\section{REPOSITORY RECORD}

Cai, R.G., and J.B. Griffiths. 2019. "Null Particle Solutions in Three-dimensional (anti-) De Sitter Spaces". figshare. https://hdl.handle.net/2134/832. 


\title{
Null particle solutions in three-dimensional (anti-) de Sitter spaces
}

\author{
Rong-Gen Cai* \\ Center for Theoretical Physics, \\ Seoul National University, \\ Seoul 151-742, Korea \\ and J. B. Griffiths ${ }^{\dagger}$ \\ Department of Mathematical Sciences \\ Loughborough University \\ Loughborough, Leics. LE11 3TU, U.K.
}

May 4, 1999

\begin{abstract}
We obtain a class of exact solutions representing null particles moving in three-dimensional (anti-) de Sitter spaces by boosting the corresponding static point source solutions given by Deser and Jackiw. In de Sitter space the resulting solution describes two null particles moving on the (circular) cosmological horizon, while in anti-de Sitter space it describes a single null particle propagating from one side of the universe to the other. We also boost the BTZ black hole solution to the ultrarelativistic limit and obtain the solution for a spinning null particle moving in anti-de Sitter space. We find that the ultrarelativistic geometry of the black hole is exactly the same as that resulting from boosting the Deser-Jackiw solution when the angular momentum of the hole vanishes. A general class of solutions is also obtained which represents several null particles propagating in the Deser-Jackiw background. The differences between the three-dimensional and four-dimensional cases are also discussed.
\end{abstract}

${ }^{*}$ E-mail: cai@wormhole.snu.ac.kr
${ }^{\dagger}$ E-mail: J.B.Griffiths@Lboro.ac.uk 


\section{Introduction}

Although the Einstein equations still hold in three-dimensional spacetime, the nature of gravity is quite different from that in four-dimensional spacetime. Because the Einstein and Riemann tensors are equivalent in three-dimensional spacetime, general relativity is dynamically trivial there. That is, the vacuum spacetime is flat. The localised sources have effects only on the global geometry. In 1984, Deser, Jackiw, and 't Hooft [1] investigated in detail the Einstein gravity with static point sources in three-dimensional spacetime. For a single static particle, the geometry is given by cutting a sector out of the Euclidean two-plane along two straight lines, and identifying the edges to form a cone. Gravity theories with lightlike sources and spacelike source in three-dimensional flat spacetime have also been analysed in [2] and [3], respectively.

When a nonvanishing cosmological constant is introduced to the three-dimensional Einstein gravity, some significant changes occur. In this case, the spacetime has constant curvature and corresponds either to de Sitter or to anti-de Sitter space. In the de Sitter space, the static two-particle solution is a sphere minus a wedge with the edges identified. This is because the twospace is a sphere in a three-dimensional covering space. To obtain the effect of a point particle one can cut the sphere from the location of the source along two great circles. On a sphere, these cuts meet again at the antipodal point. By identifying along the cuts, this procedure automatically creates a "mirror" source. There is no pure one-particle solution globally. For the anti-de Sitter case, the two-space is a hyperboloid. This can be cut along two lines and the cuts identified to produce single particle solutions. Deser and Jackiw [4] have obtained a metric (hereafter denoted the DJ solution) and confirmed the above geometrical picture for static point sources by directly solving the Einstein equations with a cosmological constant.

In this paper we investigate the Einstein gravity with null particle sources in the threedimensional de Sitter and anti-de Sitter spaces. Initially, we employ the boost method that was first used by Aichelburg and Sexl [5] to derive the gravitational field generated by a photon. By boosting the Schwarzschild solution to the ultrarelativistic limit in which the velocity of the source approaches the speed of light and the mass is scaled to zero in an appropriate manner, Aichelburg and Sexl derived a solution describing an impulsive gravitational wave propagating in a flat spacetime. This method has subsequently been widely used to investigate the gravitational fields generated by various null sources moving in flat spacetimes (for a brief review see [6] and references cited therein). Due to the fact that the four-dimensional (anti-) de Sitter space can be represented as a four-dimensional hyperboloid embedded in a five-dimensional flat spacetime, Hotta and Tanaka [7] succeeded in obtaining exact solutions for null particles moving in (anti-) de Sitter spacetimes by boosting the Schwarzschild-(anti-)de Sitter solutions. The impulsive wave surfaces generated have been discussed in detail by Podolský and Griffiths [8]. Further they considered more general gravitational wave solutions in (anti-) de Sitter spaces [9]. These can be interpreted as impulsive gravitational waves generated by an arbitrary distribution of null particles each with arbitrary multipole structure.

The plan of this paper is as follows. In the next section, we will introduce the DJ solution and boost the spacetime to the ultrarelativistic limit in the three-dimensional de Sitter and anti-de Sitter spaces and then analyse the resulting geometries. We will also boost the BañadosTeitelboim-Zanelli (BTZ) black hole [10] in the anti-de Sitter space in section 3. Although the BTZ black hole solution is quite different from the DJ solution globally, we find that, when the angular momentum of the BTZ black hole vanishes, the resulting geometries are equivalent to each other. In section 4 we will consider the null-particle solution in the DJ background, and further confirm the result derived using the boost method. A brief discussion of the main results is included in section 5 . 


\section{Boosting the DJ solutions in the (anti-) de Sitter spaces}

The three-dimensional Einstein equations with a nonvanishing cosmological constant can be written as

$$
R_{\mu \nu}-\frac{1}{2} R g_{\mu \nu}+\Lambda g_{\mu \nu}=8 \pi T_{\mu \nu}
$$

where $\Lambda$ denotes the cosmological constant and $T_{\mu \nu}$ the energy-momentum tensor of the sources. Here the gravitational constant $G$ has been set to one.

The solutions which describe a static point particle at the origin in the (anti-) de Sitter spaces were found to be [4]

$$
d s^{2}=-N^{2}(R) d t^{2}+\Phi(R)\left(d R^{2}+R^{2} d \phi^{2}\right),
$$

where

$$
\begin{aligned}
\Phi(R) & =\frac{4 \alpha^{2}}{\Lambda R^{2}\left[\left(R / R_{0}\right)^{\alpha}+\left(R / R_{0}\right)^{-\alpha}\right]^{2}}, \\
N(R) & =\frac{\left(R / R_{0}\right)^{\alpha}-\left(R / R_{0}\right)^{-\alpha}}{\left(R / R_{0}\right)^{\alpha}+\left(R / R_{0}\right)^{-\alpha}}
\end{aligned}
$$

$R_{0}$ is an integration constant and $\alpha=1-4 M$. The constant $M$ is the mass of the point particle. Performing a simple coordinate transformation in (2) gives

$$
d s^{2}=-\left(1-\Lambda r^{2} / \alpha^{2}\right) d t^{2}+\alpha^{-2}\left(1-\Lambda r^{2} / \alpha^{2}\right)^{-1} d r^{2}+r^{2} d \phi^{2} .
$$

When $\Lambda>0$, the solution (4) has a cosmological event horizon at $r_{c}=\alpha / \sqrt{\Lambda}$ with surface gravity $\kappa=\sqrt{\Lambda}$. When $\alpha=1$, that is for the vacuum case $M=0$, the DJ solution (4) reduces to the familiar form

$$
d s^{2}=-\left(1-\Lambda r^{2}\right) d t^{2}+\left(1-\Lambda r^{2}\right)^{-1} d r^{2}+r^{2} d \phi^{2}
$$

which is just the three-dimensional de Sitter $(\Lambda>0)$ or anti-de Sitter $(\Lambda<0)$ space in static coordinates.

Similar to the case in four dimensions, the three-dimensional (anti-) de Sitter space can also be represented as a hyperboloid embedded in a four-dimensional flat spacetime. Let us first consider the case of the de Sitter space.

(i). In de Sitter space. In this case, the hyperboloid satisfies

$$
-Z_{0}^{2}+Z_{1}^{2}+Z_{2}^{2}+Z_{3}^{2}=a^{2}
$$

where $a^{2}=1 / \Lambda$. The de Sitter space can be expressed as the following $S O(1,3)$ invariant line element satisfying the constraint (6)

$$
d s_{\mathrm{ds}}^{2}=-d Z_{0}^{2}+d Z_{1}^{2}+d Z_{2}^{2}+d Z_{3}^{2} .
$$

Obviously, when we parametrize the hypersurface (6) with the following coordinates

$$
\begin{aligned}
& Z_{0}=\sqrt{a^{2}-r^{2}} \sinh (t / a), \quad Z_{1}=r \cos \phi, \\
& Z_{3}= \pm \sqrt{a^{2}-r^{2}} \cosh (t / a), \quad Z_{2}=r \sin \phi,
\end{aligned}
$$

the metric (5) can be deduced from (7). When boosting the DJ solution in the de Sitter space (7), it is appropriate first to expand the solution (4) up to the first order of the mass $M$ (higher order contributions will vanish due to the boost). This yields

$$
d s^{2} \approx d s_{\mathrm{ds}}^{2}+8 M \Lambda r^{2} d t^{2}+\frac{8 M}{\left(1-\Lambda r^{2}\right)^{2}} d r^{2}
$$


where $d s_{\mathrm{ds}}^{2}$ denotes the de Sitter space (5). Using the coordinates (8), we can rewrite (9) as

$$
\begin{aligned}
d s^{2}= & d s_{\mathrm{ds}}^{2}+\frac{8 M}{\left(Z_{3}^{2}-Z_{0}^{2}\right)^{2}}\left[\left(a^{2}+Z_{0}^{2}-Z_{3}^{2}\right)\left(Z_{3} d Z_{0}-Z_{0} d Z_{3}\right)^{2}\right. \\
& \left.+\frac{a^{4}}{\left(a^{2}+Z_{0}^{2}+Z_{3}^{2}\right)}\left(Z_{3} d Z_{3}-Z_{0} d Z_{0}\right)^{2}\right] .
\end{aligned}
$$

We now make a Lorentz boost in the $Z_{1}$-direction, that is, a Lorentz transformation

$$
Z_{0} \rightarrow \frac{Z_{0}+v Z_{1}}{\sqrt{1-v^{2}}}, \quad Z_{1} \rightarrow \frac{v Z_{0}+Z_{1}}{\sqrt{1-v^{2}}}, \quad Z_{2} \rightarrow Z_{2}, \quad Z_{3} \rightarrow Z_{3}
$$

where $v$ is the boost velocity. To obtain a result of physical interest, the mass must be reduced to zero in an appropriate way. Following [5], we scale mass as

$$
M=p \sqrt{1-v^{2}}
$$

where $p$ is a constant which can be interpreted as the energy of the null particle. Substituting (11) and (12) into (10), we obtain

$$
\begin{aligned}
d s^{2}= & d s_{\mathrm{ds}}^{2}+\frac{8 p \sqrt{1-v^{2}}}{\left(Z_{3}^{2}-z^{2}\right)^{2}}\left[\left(a^{2}+z^{2}-Z_{3}^{2}\right)\left(Z_{3} d z-z d Z_{3}\right)^{2}\right. \\
& \left.+\frac{a^{4}}{\left(a^{2}+z^{2}-Z_{3}\right)}\left(Z_{3} d Z_{3}-z d z\right)^{2}\right]
\end{aligned}
$$

where $z^{2}=\left(Z_{0}+v Z_{1}\right)^{2} /\left(1-v^{2}\right)$. Using the identity

$$
\lim _{v \rightarrow 1} \frac{1}{\sqrt{1-v^{2}}} f\left(z^{2}\right)=\delta\left(Z_{0}+Z_{1}\right) \int_{-\infty}^{\infty} f\left(z^{2}\right) d z
$$

and taking the limit $v \rightarrow 1$ in (13), we obtain

$$
d s^{2}=d s_{\mathrm{ds}}^{2}-8 \pi p\left|Z_{2}\right| \delta\left(Z_{0}+Z_{1}\right)\left(d Z_{0}+d Z_{1}\right)^{2} .
$$

This looks like an impulsive wave solution in the de Sitter space, located on the surface $Z_{0}+Z_{1}=$ $0, Z_{2}^{2}+Z_{3}^{2}=a^{2}$ which at any time is a circle of constant radius.

In order to further analyse this solution, it proves convenient to use the following coordinates $[8]$

$$
\begin{array}{ll}
Z_{0}=\frac{1}{2 \eta}\left[a^{2}-\eta^{2}+(x-a)^{2}+y^{2}\right], & Z_{1}=\frac{a}{\eta}(x-a), \\
Z_{3}=\frac{1}{2 \eta}\left[a^{2}+\eta^{2}-(x-a)^{2}-y^{2}\right], & Z_{2}=\frac{a}{\eta} y
\end{array}
$$

Further we can put

$$
x=\rho \cos \phi, \quad y=\rho \sin \phi,
$$

with $\rho \in[0, \infty), \phi \in[0,2 \pi)$. The de Sitter space can then be described as

$$
d s_{\mathrm{ds}}^{2}=\frac{a^{2}}{\eta^{2}}\left(-d \eta^{2}+d \rho^{2}+\rho^{2} d \phi^{2}\right),
$$

which is in conformally flat form. The solution (15) can then be rewritten as

$$
d s^{2}=d s_{\mathrm{ds}}^{2}-8 \pi p a|\sin \phi|\left[\delta(\eta-\rho)(d \eta-d \rho)^{2}+\delta(\eta+\rho)(d \eta+d \rho)^{2}\right] .
$$


This looks like two impulsive wavefronts. However, as pointed out in [8] in the four-dimensional case, both components are required for the conformal picture to be geodesically complete. Because $\rho \geq 0$, the term $\delta(\eta-\rho)$ does not vanish for $\eta \geq 0$ only, while $\delta(\eta+\rho)$ is required for $\eta \leq 0$. From (19), it is clear that the particles are located on the circle $\rho=|\eta|$ which is the cosmological horizon of the de Sitter space.

It can then be shown that the energy-momentum tensor is only non-zero at the two points $Z_{0}+Z_{1}=0, Z_{2}=0, Z_{3}= \pm a$ which thus represent two null particles. At all other points on this null surface, the impulsive component can in fact be removed by a discontinuous coordinate transformation. The solution can thus be represented as a three-dimensional de Sitter space cut along the cosmological horizon $Z_{0}+Z_{1}=0$, with the two halves reattached in such a way as to create two null particles at the points $Z_{2}=0$, or $y=0$, or $\phi=0, \pi$, which are at opposite points on the horizon. This situation is very like that in the four-dimensional case, in which instead of the circle the wave surface is spherical and the particles are located at opposite poles. The significant difference, however, is that in the four-dimensional case the Weyl tensor has some non-zero components on the spherical surface and these can be interpreted as describing gravitational wave components generated by the null particles. In the three-dimensional theory such free gravitational waves cannot occur.

(ii). In anti-de Sitter space. We now turn to the case of the three-dimensional anti-de Sitter space. This can be regarded as a hyperboloid

$$
-Z_{0}^{2}+Z_{1}^{2}+Z_{2}^{2}-Z_{3}^{2}=-a^{2}
$$

embedded in an $S O(2,2)$ invariant four-dimensional flat spacetime

$$
d s_{\text {ads }}^{2}=-d Z_{0}^{2}+d Z_{1}^{2}+d Z_{2}^{2}-d Z_{3}^{2},
$$

where $a^{2}=-1 / \Lambda>0$. Obviously, the anti-de Sitter space (5) can be parametrized by the following coordinates

$$
\begin{array}{ll}
Z_{0}=\sqrt{a^{2}+r^{2}} \sin (t / a), & Z_{1}=r \cos \phi, \\
Z_{3}=\sqrt{a^{2}+r^{2}} \cos (t / a), & Z_{2}=r \sin \phi .
\end{array}
$$

We now boost the DJ solution in the anti-de Sitter space. Again expanding the solution up to the first order in the mass $M$ and using the coordinates (22), we arrive at

$$
\begin{aligned}
d s^{2}= & d s_{\text {ads }}^{2}+\frac{8 M}{\left(Z_{0}^{2}+Z_{3}^{2}\right)^{2}}\left[\left(Z_{0}^{2}+Z_{3}^{2}-a^{2}\right)\left(Z_{3} d Z_{0}-Z_{0} d Z_{3}\right)^{2}\right. \\
& \left.+\frac{a^{4}}{\left(Z_{0}^{2}+Z_{3}^{2}-a^{2}\right)}\left(Z_{3} d Z_{3}-Z_{0} d Z_{0}\right)^{2}\right] .
\end{aligned}
$$

Repeating the same steps as in the case of the de Sitter space, that is, using the Lorentz transformation (11), rescaling the mass as (12), and taking the limit $v \rightarrow 1$, finally we can obtain

$$
d s^{2}=d s_{\text {ads }}^{2}-8 \pi p\left|Z_{2}\right| \delta\left(Z_{0}+Z_{1}\right)\left(d Z_{0}+d Z_{1}\right)^{2} .
$$

Comparing with (15), it is easy to see that the expression for the apparent impulsive part is the same as that in the de Sitter space. However, the interpretation is quite different. In this case the impulsive component is given by the surface $Z_{0}+Z_{1}=0, Z_{2}^{2}-Z_{3}^{2}=-a^{2}$ which at any time is a hyperbola. Let us now analyse this solution.

In the anti-de Sitter space, introduce first the following coordinates

$$
\begin{array}{ll}
Z_{0}=\frac{1}{2 x}\left[a^{2}-\eta^{2}+x^{2}+(y-a)^{2}\right], & Z_{1}=\frac{a}{x}(y-a), \\
Z_{2}=\frac{1}{2 x}\left[a^{2}+\eta^{2}-x^{2}-(y-a)^{2}\right], & Z_{3}=\frac{a}{x} \eta,
\end{array}
$$


and then use

$$
x=\rho \cos \phi, \quad y=\rho \sin \phi .
$$

This produces the anti-de Sitter space written in the conformally flat form

$$
d s_{\text {ads }}^{2}=\frac{a^{2}}{\rho^{2} \cos ^{2} \phi}\left[-d \eta^{2}+d \rho^{2}+\rho^{2} d \phi^{2}\right] .
$$

The solution (24) can be rewritten in these coordinates as

$$
d s^{2}=d s_{\text {ads }}^{2}-\frac{8 \pi p a|\sin \phi|}{\cos ^{2} \phi}\left[\delta(\eta-\rho)(d \eta-d \rho)^{2}+\delta(\eta+\rho)(d \eta+d \rho)^{2}\right] .
$$

Here it should be stressed that the solution (28) does not mean two impulses again. As in the de Sitter case, $\delta(\eta-\rho)$ works only for $\eta>0$ while $\delta(\eta+\rho)$ for $\eta<0$. The two components are required for globally geodesic completeness. From (28), it is clear that the impulsive component is located on the line $\rho=|\eta|$, or $x^{2}+y^{2}=\eta^{2}$. However, this is not a circle - according to (27) it is conformal to a circle, and the coordinate $\phi$ is restricted to $-\pi / 2<\phi<\pi / 2$. In fact it is a hyperbola. It can then be shown that this solution represents a single null particle located at $Z_{2}=0$ on the null surface $Z_{0}+Z_{1}=0$, i.e. at $y=0, x=\eta($ or $x=-\eta)$. The particle thus clearly propagates from one side of the universe to the other and (since this spacetime contains closed timelike lines) may then be considered to propagate back in the opposite direction.

Thus, by boosting the DJ solutions, we have obtained two kinds of exact solutions describing null particles moving in the three-dimensional de Sitter and anti-de Sitter spaces. (In section 4 we will further confirm these results by directly solving the Einstein equations.) Although static particles only have effect on the global geometry, which is quite different from the situation in the four-dimensional case, we still find that the boost method is sufficiently powerful to derive null particle solutions from their corresponding static particle solutions. In the next section we will boost the BTZ black hole solution in the anti-de Sitter space. In the static situation, this is quite different from the DJ solution from the aspect of global properties. However, the resulting ultrarelativistic geometry is found to be identical, at least in the non-rotating case.

\section{Boosting the BTZ black hole solution in the anti-de Sitter space}

Due to the special properties of three-dimensional gravity, it was a surprising discovery when Bañados, Teitelboim, and Zanelli [10] claimed that they found a black hole solution in the Einstein gravity with a negative cosmological constant. The solution they found is

$$
d s^{2}=-N^{2}(r) d t^{2}+N^{-2} d r^{2}+r^{2}\left(N^{\phi}(r) d t+d \phi\right)^{2},
$$

where

$$
N^{2}=-8 M+\frac{r^{2}}{a^{2}}+\frac{16 J^{2}}{r^{2}}, \quad N^{\phi}=-\frac{4 J}{r^{2}} .
$$

Here $-1 / a^{2}$ denotes the negative cosmological constant. The integration constants $M$ and $J$ can be interpreted as the mass and angular momentum of the black hole. This black hole has two horizons at

$$
r_{ \pm}^{2}=4 M a^{2}\left[1 \pm \sqrt{1-\left(\frac{J}{M a}\right)^{2}}\right]
$$

provided $M>0$ and $J<M a$. This solution is asymptotically an anti-de Sitter spacetime and can be constructed by identifying some discrete points in the three-dimensional anti-de Sitter 
space. It is of interest to note, however, that when the mass and angular momentum of the hole vanish, the solution does not reduce to the anti-de Sitter space. Rather, the anti-de Sitter spacetime (5) can only be obtained from (29) in the limit as $8 M \rightarrow-1$ and $J \rightarrow 0$.

Before boosting the BTZ solution, it is first appropriate to expand it about the background of the anti-de Sitter space. To achieve this, we expand the BTZ solution (29) to first order in the mass term $8 M+1$ and the angular momentum $J$. The result is

$$
d s^{2} \approx d s_{\text {ads }}^{2}+(8 M+1) d t^{2}+\frac{8 M+1}{\left(1+r^{2} / a^{2}\right)^{2}} d r^{2}-8 J d t d \phi .
$$

Using the coordinates in (22), the above metric can be rewritten as

$$
\begin{aligned}
d s^{2}= & d s_{\text {ads }}^{2}+\frac{(8 M+1) a^{2}}{\left(Z_{0}^{2}+Z_{3}^{2}\right)^{2}}\left[\left(Z_{3} d Z_{0}-Z_{0} d Z_{3}\right)^{2}+\frac{a^{2}}{\left.Z_{3}^{2}+Z_{0}^{2}-a^{2}\right)}\left(Z_{3} d Z_{3}+Z_{0} d Z_{0}\right)^{2}\right] \\
& -\frac{8 J a}{\left(Z_{3}^{2}+Z_{0}^{2}\right)\left(Z_{3}^{2}+Z_{0}^{2}-a^{2}\right)}\left(Z_{3} d Z_{0}-Z_{0} d Z_{3}\right)\left(Z_{1} d Z_{2}-Z_{2} d Z_{1}\right) .
\end{aligned}
$$

We now make a Lorentz boost (11) in the $Z_{1}$-direction, rescaling the mass and angular momentum as

$$
8 M+1=8 p \sqrt{1-v^{2}}, \text { and } J=s \sqrt{1-v^{2}} .
$$

We then proceed to the ultrarelativistic limit $v \rightarrow 1$. In this case, the two constants $p$ and $s$ can be interpreted physically as the energy and spin angular momentum of the resulting null particle respectively. It may be observed that, in this limit, the inequality $J<M a$ mentioned above is strictly violated. This is because the mass $M$ and angular momentum $J$ are rescaled in different ways. However, the limit is still an exact solution even though it is not strictly the limit of a real rotating black hole. Using this procedure in (33), we obtain

$$
\begin{aligned}
d s^{2}=\quad & d s_{\text {ads }}^{2}+8 \pi p\left(Z_{3}-\sqrt{Z_{3}-a^{2}}\right) \delta\left(Z_{0}+Z_{1}\right)\left(d Z_{0}+d Z_{1}\right)^{2} \\
& -\frac{8 \pi s}{a}\left[Z_{2}-\frac{Z_{2} Z_{3}}{\sqrt{Z_{3}^{2}-a^{2}}}\right] \delta\left(Z_{0}+Z_{1}\right)\left(d Z_{0}+d Z_{1}\right)^{2} .
\end{aligned}
$$

The two linear terms in the solution (35) can be removed by the following discontinuous linear transformation

$$
\begin{aligned}
& Z_{2} \rightarrow Z_{2}-\frac{4 \pi s}{a} U \Theta(U) \\
& Z_{3} \rightarrow Z_{3}-4 \pi p U \Theta(U) \\
& U \rightarrow U \\
& V \rightarrow V-16 \pi^{2} p^{2} U \Theta(U)+8 \pi p Z_{3} \Theta(U)+\frac{16 \pi^{2} s^{2}}{a^{2}} U \Theta(U)-\frac{8 \pi s}{a} Z_{2} \Theta(U),
\end{aligned}
$$

where $U=Z_{0}+Z_{1}, V=Z_{0}-Z_{1}$, and $\Theta$ is the Heaviside step function. Therefore, the solution (35) can be reduced to

$$
d s^{2}=d s_{\text {ads }}^{2}-8 \pi p\left|Z_{2}\right| \delta\left(Z_{0}+Z_{1}\right)\left(d Z_{0}+d Z_{1}\right)^{2}+\frac{8 \pi s Z_{3}}{a} \operatorname{sign}\left(Z_{2}\right) \delta\left(Z_{0}+Z_{1}\right)\left(d Z_{0}+d Z_{1}\right)^{2}
$$

It is now easy to see that when $s=0$, that is when the angular momentum vanishes in the original BTZ solution, the solution (37) is identical to (24). Thus, both ultrarelativistic limits of the DJ solution for $\Lambda<0$ and the spinless BTZ solution are equivalent to each other. Obviously, 
the third term in (37) is the spin effect of the null particle. In the coordinates (25) and (26), we can rewrite $(37)$ as

$$
\begin{aligned}
d s^{2}= & d s_{\text {ads }}^{2}+\left(-8 \pi p a \frac{|\sin \phi|}{\cos ^{2} \phi}+\frac{8 \pi s \operatorname{sign}(\tan \phi)}{\cos \phi|\cos \phi|}\right)\left[\delta(\eta-\rho)(d \eta-d \rho)^{2}\right. \\
& \left.+\delta(\eta+\rho)(d \eta+d \rho)^{2}\right] \\
= & d s_{\text {ads }}^{2}+\left(-8 \pi p a \frac{|\sin \phi|}{\cos ^{2} \phi}+\frac{8 \pi s}{\cos ^{2} \phi} \operatorname{sign}(\sin \phi)\right)\left[\delta(\eta-\rho)(d \eta-d \rho)^{2}\right. \\
& \left.+\delta(\eta+\rho)(d \eta+d \rho)^{2}\right]
\end{aligned}
$$

which is clearly identical to (28) when $s=0$.

\section{Null particles in the DJ background}

In this section we will directly solve the Einstein equations with null particle sources and reobtain some of the results of previous sections that were derived by the boost method. In [11] Dray and 't Hooft considered a particle moving with the speed of light on the Schwarzschild black hole horizon, and investigated the back reaction of the particle on the geometry. In this case, the particle produces an impulsive gravitational wave located on the Schwarzschild horizon. Loustó and Sánchez [12] and Sfetsos [13] further extended the work of Dray and 't Hooft to nonvacuum backgrounds and investigated the conditions that should be satisfied when an impulsive wave is introduced into curved spacetimes. The null particle solution in the BTZ background (29) has already been considered in [13].

Here we first note that the DJ solution (4) can be rewritten, after rescaling the coordinate $r$, as

$$
d s^{2}=-\left(1-\Lambda r^{2}\right) d t^{2}+\left(1-\Lambda r^{2}\right)^{-1} d r^{2}+\alpha^{2} r^{2} d \phi^{2} .
$$

Further defining $\phi^{\prime}=\alpha \phi$ with $\phi^{\prime} \in[0,2 \pi \alpha)$, we have

$$
d s^{2}=-\left(1-\Lambda r^{2}\right) d t^{2}+\left(1-\Lambda r^{2}\right)^{-1} d r^{2}+r^{2} d \phi^{2},
$$

which is obviously equivalent to the de Sitter space locally, but has a deficit angle $\delta=(1-\alpha) 2 \pi$. We will first discuss solutions with null particles located on the cosmological horizon of the de Sitter case $\left(\Lambda=1 / a^{2}>0\right)$. The metric (39) or (40) also can be regarded as a hypersurface embedded in the flat spacetime $(7)$ with

$$
\begin{aligned}
& Z_{0}=\sqrt{a^{2}-r^{2}} \sinh (t / a), \quad Z_{1}=r \cos (\alpha \phi), \\
& Z_{3}= \pm \sqrt{a^{2}-r^{2}} \cosh (t / a), \quad Z_{2}=r \sin (\alpha \phi) .
\end{aligned}
$$

Introducing the null coordinates

$$
u=e^{t / a} F(r), \quad v=e^{-t / a} F(r),
$$

where the function $F(r)$ is defined as

$$
F(r) \equiv \exp \left(-\frac{1}{a} \int \frac{d r}{\left(1-r^{2} / a^{2}\right)}\right)=\left(\frac{a-r}{a+r}\right)^{1 / 2},
$$

we can re-express the DJ solution (40) as

$$
d s^{2}=2 A(u, v) d u d v+r^{2}(u, v) d \phi^{\prime 2},
$$


where

$$
A(u, v)=\frac{\left(a^{2}-r^{2}\right)}{2 F^{2}(r)}, \quad r(u, v)=\frac{a(1-u v)}{1+u v} .
$$

We can now consider the effect of null particles located on the null surface $u=0$ which is clearly the cosmological horizon $r=a$. In this three-dimensional theory, this horizon is circular. Following [11] and [13], we can adopt the coordinate shift method on the background (40). That is, the following ansatz is employed: For $u<0$ the spacetime is still the background (44) while for $u>0$ the spacetime is (44) with $v$ shifted as $v \rightarrow v+f\left(\phi^{\prime}\right)$. The function $f\left(\phi^{\prime}\right)$ which will be determined later describes the effect of the sources. Using this approach, the new solution has the form

$$
d s^{2}=2 A(u, v) d u d v-2 A(u, v) f\left(\phi^{\prime}\right) \delta(u) d u^{2}+r^{2}(u, v) d \phi^{\prime 2},
$$

which comes from (44) after making the coordinate shift:

$$
u \rightarrow u, \quad v \rightarrow v-f\left(\phi^{\prime}\right) \Theta(u), \quad \phi^{\prime} \rightarrow \phi^{\prime} .
$$

For this solution to be consistent with the Einstein equations, the following conditions must be satisfied at $u=0$ [13],

$$
\begin{aligned}
& A_{, v}=r_{, v}^{2}=T_{v v}=0 \\
& \frac{d^{2} f}{d \phi^{\prime 2}}-\frac{r_{, u v}^{2}}{2 A} f=\frac{8 \pi r^{2}}{A} \tilde{T}_{u u}
\end{aligned}
$$

where $T$ is the energy-momentum tensor of matter generating the DJ geometry, that is, the cosmological constant and possibly some static point particles, and $\tilde{T}$ is the energy-momentum tensor of any null particles located on the surface. Here it should be noticed that the only nonvanishing component of the energy-momentum tensor for null particles is $\tilde{T}_{u u}$, and that this is zero everywhere except at the points where the particles are located.

At the $u=0$ null surface - that is, on the cosmological horizon $r_{c}=a=1 / \sqrt{\Lambda}$ for the DJ $(\Lambda>0)$ solution $(40)$ - it is easy to see that the conditions (48) are satisfied and

$$
\left.A(u, v)\right|_{u=0}=2 a^{2},\left.\quad r_{, u v}^{2}\right|_{u=0}=-4 a^{2} .
$$

Then (49) reduces to

$$
\frac{d^{2} f}{d \phi^{\prime 2}}+f=4 \pi \tilde{T}_{u u}
$$

It may immediately be observed that a solution with $f=4 \pi \rho$, where $\rho$ is a constant, represents a uniform distribution of null matter (of density $\rho$ ) over the circular horizon. Since equation (51) is linear, this component can always be added to other components. However, we will ignore this possibility in the remainder of this section.

In those parts of the null surface on which $\tilde{T}_{u u}=0$, equation (51) has the solution

$$
f=c \sin \left(\phi^{\prime}+\omega^{\prime}\right)=c \sin \alpha(\phi+\omega)
$$

where $c$ and $\omega^{\prime}=\alpha \omega$ are arbitrary constants. This solution for $f$ around the circular horizon can always be removed by a discontinuous coordinate transformation. However, solutions describing several discrete particles can be constructed by patching different sections of the sine wave, each with different amplitude and phase. Points at which $f$ is $C^{0}$ but has a discontinuous first derivative can be interpreted as points at which null particles are located. The energy of each particle is then represented by the jump in the derivative of $f$, and the energy-momentum tensor $\tilde{T}_{u u}$ is given by a $\delta$-function. On considering (38), it may be observed that discontinuities 
in $f$ may also be permitted. These represent point particles with spin and, in this case, the energy-momentum tensor $\tilde{T}_{u u}$ contains a derivative of a $\delta$-function.

For example, consider the case in which $n$ particles each of energy $p_{i}, i=1 \ldots n$, are located at points $\phi=\phi_{i}$ around the circular wave. The solution is then given by

$$
f=c_{i} \sin \alpha\left(\phi+\omega_{i}\right) \quad \text { for } \quad \phi_{i-1} \leq \phi \leq \phi_{i}
$$

where $n+1 \rightarrow 1$. It is then possible to choose the $2 n$ arbitrary constant $c_{i}$ and $\omega_{i}$ such that

$$
\begin{aligned}
& c_{i+1} \sin \alpha\left(\phi_{i}+\omega_{i+1}\right)-c_{i} \sin \alpha\left(\phi_{i}+\omega_{i}\right)=0 \\
& c_{i+1} \cos \alpha\left(\phi_{i}+\omega_{i+1}\right)-c_{i} \cos \alpha\left(\phi_{i}+\omega_{i}\right)=\frac{4 \pi p_{i}}{a}
\end{aligned}
$$

By choosing the constants appropriately, it is possible to construct solutions in which $n(\geq 2)$ null particles of arbitrary energy are distributed arbitrarily round the circular wave.

In particular, we can consider the two-particle solution in which the particles are located at opposite ends of a diameter of the circle. Since $0 \leq \phi^{\prime}<2 \pi \alpha$ around the circle, we may consider the particles to be located at points given by $\phi^{\prime}=0$ and $\phi^{\prime}=\pi \alpha$. We may also restrict attention to the case in which the two particles have identical energy $p$. Such a solution can be constructed by the above method in which

$$
c_{1}=c_{2}=\frac{2 \pi p}{a} \operatorname{cosec} \frac{\pi \alpha}{2}, \quad \omega_{1}=\frac{(1-\alpha) \pi}{2 \alpha}, \quad \omega_{2}=\frac{(1-3 \alpha) \pi}{2 \alpha} .
$$

This solution represents two null particles propagating in the DJ $(\Lambda>0)$ background. In the case in which $\alpha=1$, the background is the de Sitter space and the solution can alternatively be written in the form

$$
f=\frac{2 \pi p}{a}|\sin \phi|
$$

This is clearly identical (after some rescaling) to the solution (19) that was obtained by boosting two static particles in the de Sitter background, and thus confirms this solution.

It may also be observed that, in the particular case in which $\alpha=1 / 2$, the deficit angle in $\phi^{\prime}$ is $\pi$ and a one-particle solution at $\phi=0$ can easily be constructed using

$$
f=c \sin (\phi / 2) \quad \text { where } \quad 0 \leq \phi \leq 2 \pi .
$$

It is also possible to obtain solutions for null particles propagating in the DJ background with $\Lambda<0$. However, the Dray-'t Hooft [11] method cannot be directly used in this case. Nevertheless, equivalent equations can be obtained and these will include, for $\alpha=1$, the special cases (28) of a null particle and (38) of a spinning null particle propagating in an anti-de Sitter space.

\section{Conclusion and discussion}

We have investigated null particle solutions in the three-dimensional de Sitter and anti-de Sitter spaces by boosting the corresponding static point source solutions (DJ solutions) [4] in the (anti-) de Sitter backgrounds. For the de Sitter case, the resulting solution describes two null particles located at opposite points on the cosmological horizon which forms a circle of constant size. For the anti-de Sitter case, the solution describes a single null particle located at the point of symmetry of a propagating hyperbola. We have also boosted the BTZ black hole solution to the ultrarelativistic limit. Although the BTZ black hole is quite different from the DJ solution globally, we have found that these two ultrarelativistic limits are equivalent to each other when the angular momentum of the hole is zero. This means that the boost method may loose some 
memory of the original solution in the process of the boost. In addition, we believe that the angular momentum of the hole gives the spin effect of the corresponding null particle.

By using the coordinate shift method, we have also obtained null particle solutions in DJ background. When $\alpha=1$, the DJ $(\Lambda>0)$ solution reduces to the de Sitter space, and the results obtained include that derived using the boost method. It may be observed that the boost method indeed is very powerful in the derivation of null particle solutions, not only in flat spacetime, but also in the (anti-) de Sitter space in three dimensions as well as four.

Due to the special properties of the geometry in three-dimensional spacetime, the nature of Einstein gravity is rather different from that in four dimensions. The static point source solutions, given in [1] in flat spacetime and in [4] in (anti-) de Sitter space, clearly demonstrate the differences in the local and global aspects from the four-dimensional Schwarzschild and Schwarzschild-(anti-)de Sitter solutions. By comparing the null particle solution given by Deser and Steif [2] in three-dimensional flat spacetime and some results given in this paper, we may observe some similarities as well as some differences in the null particle solutions in three and four dimensions. The main difference in four-dimensional spacetime is that the null particles generate impulsive gravitational waves which are forbidden in three-dimensional theories. It is of some interest to further compare spacelike source solutions in three and four-dimensional spacetimes. Furthermore, it also might be interesting to discuss the geodesics and particle scattering in the null particle solutions in the (anti-) de Sitter space.

\section{References}

[1] S. Deser, R. Jackiw and G. 't Hooft, Ann. Phys. 152, 220 (1984).

[2] S. Deser and A. R. Steif, Class. Quantum Grav. 9, L153 (1992).

[3] S. Deser, R. Jackiw and G. 't Hooft, Phys. Rev. Lett. 68, 267 (1992)

[4] S. Deser and R. Jackiw, Ann. Phys. 153, 405 (1984).

[5] P. C. Aichelburg and R. U. Sexl, Gen. Rel. Grav. 2, 303 (1971).

[6] R. G. Cai, J. Y. Ji and K. S. Soh, Nucl. Phys. B 528, 265 (1998).

[7] M. Hotta and M. Tanaka, Class. Quantum Grav. 10, 307 (1993).

[8] J. Podolský and J. B. Griffiths, Phys. Rev. D 56, 4756 (1997).

[9] J. Podolský and J. B. Griffiths, Class. Quantum Grav. 15, 453 (1998).

[10] M. Bañados, C. Teitelboim and J. Zanelli, Phys. Rev. Lett. 69, 1849 (1992).

M. Bañados, M. Henneaux, C. Teitelboim and J. Zanelli, Phys. Rev. D 48, 1506 (1993).

[11] T. Dray and G. 't Hooft, Nucl. Phys. B 253, 173 (1985).

[12] C. O. Loustó and N. Sánchez, Phys. Lett. B 220, 55 (1989).

[13] K. Sfetsos, Nucl. Phys. B 436, 721 (1995). 